Check for updates

Cite this: RSC Adv., 2017, 7, 46118

Received 2nd August 2017

Accepted 22nd September 2017

DOI: $10.1039 / c 7 r a 08527 c$

rsc.li/rsc-advances

\title{
Orientation-dependent induced-charge electrophoresis of magnetic metal-coated Janus particles with different coating thicknesses $\uparrow$
}

\begin{abstract}
Chia-Hsien Lin, Yu-Liang Chen (D) and Hong-Ren Jiang*
Orientation-dependent induced-charge electrophoresis (ICEP) behaviors are measured in magnetic metalcoated Janus particles, where the orientation of the particle with respect to the electric field is controlled by an external magnetic field. For a thin metallic coating $(10 \mathrm{~nm})$, the particle mainly moves normal to the electric field without the magnetic field. The velocities of ICEP in different directions are measured under the magnetic field and clear orientation-dependent velocities are observed. However, for a thick metallic coating $(70 \mathrm{~nm})$, the particle mainly moves parallel to the electric field without the magnetic field and shows an opposite tendency for orientation-dependent velocities compared with the thin metallic coated particles. We propose that the strength of the induced dipole of a Janus particle depends on the thickness of the metallic coating, which results in different orientation-dependent velocities of the Janus particle under electric fields.
\end{abstract}

\section{Introduction}

Active particles, which are known as particles that can convert energy from the environment into self-propulsion, arouse interest among scientists because of their interesting nonequilibrium transport phenomena ${ }^{1-3}$ and potential applications, such as micro-swimmers and use in drug delivery.,5 Especially, electrokinetics is widely used to drive flows and manipulate colloids, including active particles, due to its accuracy and flexibility. ${ }^{6-11}$ Bazant et al. describe the flows resulting from the action of an applied electric field on its own induced charges around a polarizable structure as "inducedcharge electro-osmosis" (ICEO), which is capable of driving Janus particles, whose surfaces have two or more distinct properties, ${ }^{12}$ under electric fields by "induced-charge electrophoresis" (ICEP). ${ }^{3,13-16}$ Janus particles driven by ICEP and the flow patterns of ICEO around the particles have been shown in different studies and become a promising field for designing small active components for different applications.,.$^{317-20}$ However, the directly experimental data of particle velocity for ICEP and flow velocity for ICEO in orientation-dependence with respect to electric fields are seldom investigated by scientists. It would be useful if the properties of ICEP and ICEO can be dynamically tuned by orientations of induced dipole on polarizable objects. Recently, several studies suggest that the motion

Institute of Applied Mechanics, National Taiwan University, No.1, Sec. 4, Roosevelt Rd., Da'an Dist., Taipei City 106, Taiwan, Republic of China.E-mail: hrjiang@iam. ntu.edu.tw; d01543004@ntu.edu.tw

$\dagger$ Electronic supplementary information (ESI) available. See DOI: $10.1039 / \mathrm{c} 7 \mathrm{ra08527c}$ of magnetic Janus particles can be manipulated by coupling an external magnetic field and the other physical field.21-25 We propose that it should be possible to control the orientation of magnetic Janus particles under an electric field by applying an external magnetic field. In this study, we measure the orientation-dependent ICEP of Janus particles with different thicknesses of coating by applying both a parallel-coplanar electric field and a magnetic field. The orientation-dependent velocity of Janus particles in electric fields is experimentally observed.

\section{Experiment}

To prepare magnetic metal-coated Janus particles, a monolayer of $3 \mu \mathrm{m}$ superparamagnetic polystyrene (PS) particles containing iron oxide nanoparticles (cat\#86055, Polysciences) is prepared by a drying process (solvent evaporation) on the glass slide which is set on a static magnet, as shown in Fig. S1. $\left.\right|^{26}$ Because of the close packing of particles, one hemisphere of the particle is shaded, and only the other hemisphere is coated with metal. ${ }^{27,28}$ The magnetic dipole of a superparamagnetic PS particle can be aligned by the static magnet. Subsequently, the monolayer is putted into a sputter (JEOL, JFC-1100E) to coat with gold. Thus, the polarity of magnetic Janus particles is determined, which is $\mathrm{N}$ pole on the gold coated hemisphere and $\mathrm{S}$ pole on the dielectric side, as shown in Fig. 1(a). The sputtered gold thickness of Janus particles measured by an atomic force microscope (AFM) is from $10 \mathrm{~nm}$ to $75 \mathrm{~nm}$ depending on the sputtering time. A chamber containing a solution and Janus particles is sandwiched by a coverslip (top) and a pair of parallel-coplanar platinum electrodes (bottom) with 

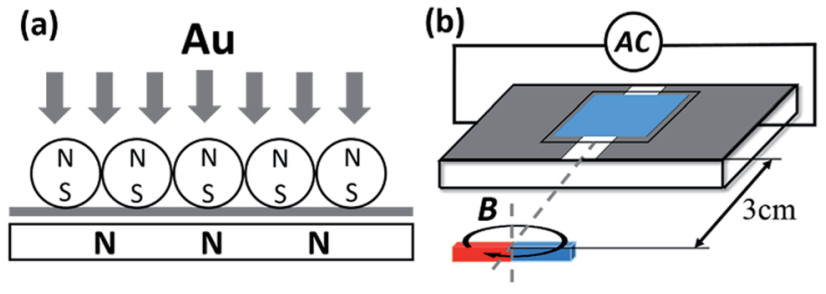

(c)

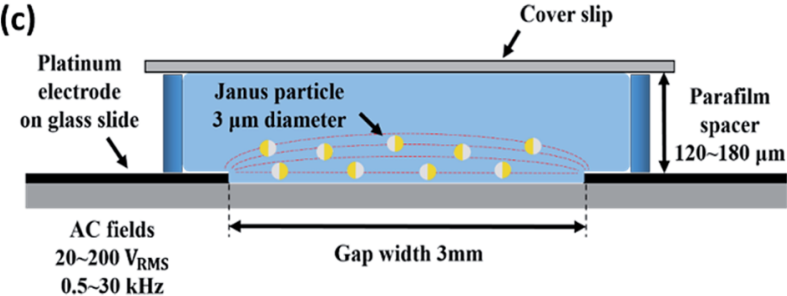

Fig. 1 (a) To prepare magnetic Janus particles, the magnetic dipoles on the $3 \mu \mathrm{m}$ superparamagnetic PS particles are aligned with $\mathrm{S}$ pole downward by an external magnetic field. After aligning, the monolayer of PS particles is coated with gold. (b) Illustration of the experimental apparatus. To generate a uniform AC electric field, a pair of electrodes are connected to a function generation and high voltage amplifier. The orientation studies are achieved by a permanent bipolar magnet installed on a step motor, which is $3 \mathrm{~cm}$ far from the chamber. (c) Side view of the experimental chamber. The particle suspension is placed in the chamber with 120-180 $\mu \mathrm{m}$ thickness, which consists of a pair of platinum electrode and a coverslip. The distance between the electrodes is $3 \mathrm{~mm}$.

a $120-180 \mu \mathrm{m}$ spacer. The platinum electrodes are fabricated by sputtering. A tape with $3 \mathrm{~mm}$ width is attached on a glass slide and this slide is putted into the sputter to coat platinum for $200 \mathrm{~s}$. The conductive layer (platinum) coated on the side measured by an AFM is about $35 \mathrm{~nm}$. After coating process is completed, the tape is removed to form a $3 \mathrm{~mm}$ gap between a pair of electrodes. The electrodes are connected to a function generator and high voltage amplifier to apply a uniform AC electric field, the voltage of which varies from 20 to $200 V_{\mathrm{RMS}}$ and the frequency is from 0.5 to $30 \mathrm{kHz}$, Fig. 1(b) and (c). A magnet installed on a step motor is used to provide a magnetic field $(<10 \mathrm{mT})$ in the arbitrary direction for manipulating the orientation of particles, Fig. 1(b). The two-dimensional trajectories of particles in the chamber are recorded by a CCD camera under an Olympus IX-70 inverted optical microscope through an object lens $(20 \times$, NA 0.5$)$.

\section{Results and discussion}

\subsection{Thickness-dependency}

To verify the coating thickness-dependent ICEP behavior of Janus particles, the velocities of magnetic Janus particles with different coating thicknesses are experimentally measured under different strength of the electric field. The ICEP velocity increases with the square of the field strength, which is consistent with the previous studies, ${ }^{3,18,20} \mathrm{Fig}$. 2 . In the case of Janus particles with a thin coating (10-25 nm), the ICEP motion of Janus particles is normal to the electric field with the dielectric hemisphere forward because of this orientation results in the largest induced dipole moment, which is aligned

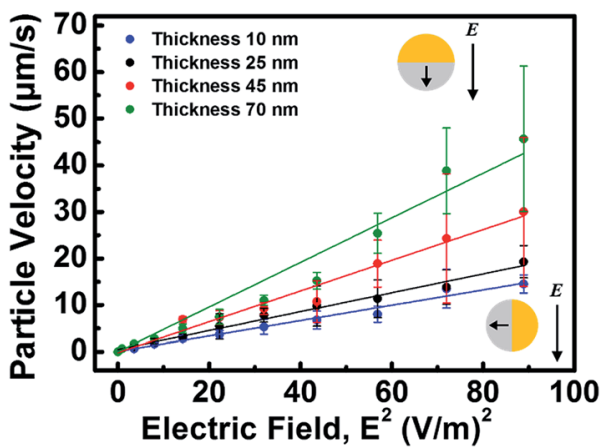

Fig. 2 Velocity of the magnetic Janus particles with different coating thicknesses varies with the squared of electric field strength at $1 \mathrm{kHz}$. Insets: (bottom) for a thin metallic coating (10-25 nm), the particle mainly moves normal to the electric field. (Top) for a thick metallic coating $(45-70 \mathrm{~nm})$, the motion of the particle becomes parallel to the electric field.

in the direction of the electric field. ${ }^{3}$ However, by increasing the coating thickness of Janus particles $(45-70 \mathrm{~nm})$, the ICEP motion becomes parallel to the electric field and the velocity also increasing with the coating thickness, as show in Fig. 2 . This result indicates that the coating thickness dominates ICEP behavior resulting in different orientations of the ICEP motion. The velocities of Janus particles are also measured under distinct field frequencies. The velocities of Janus particles decrease with the frequency (ICEP motion) and even reverse at the high frequency region $(>2 \mathrm{kHz})$, which is in accordance with previous studies (see ESI Fig. S2 $\dagger$ ). ${ }^{18,19}$

\subsection{Orientation-dependency}

To investigate the orientation-dependent ICEP behavior of Janus particles, the velocities of magnetic Janus particles with different coating thicknesses are experimentally measured in the distinct azimuthal angle from $0^{\circ}$ to $90^{\circ}$ at field frequency of $1 \mathrm{kHz}$. When the polarity of the Janus particle directed from the Au side to the dielectric side is normal (parallel) to the direction of the electric field, the azimuthal angle is defined as $0^{\circ}\left(90^{\circ}\right)$, the left inset of Fig. 3(a). In general, the ICEP motion of Janus particles is normal to the electric field with the dielectric hemisphere forward because of this orientation results in the largest induced dipole moment, which is aligned in the direction of the electric field. ${ }^{3}$ In our experiments, the orientations of magnetic Janus particles are controlled by the external magnetic field and the magnetic torque acting on the particles is given by,

$$
\tau=m_{\text {particle }} \times B
$$

where magnetic moment is $m_{\text {particle }}=\rho V\left(M_{0}+\chi_{\text {particle }} B / \mu_{0}\right), B$ is the strength of magnetic field, $\rho$ is the density of the particle, $V$ is the volume of the particle, $M_{0}$ is the initial magnetization, $\chi_{\text {particle }}$ is the initial magnetic susceptibility of the particle and $\mu_{0}$ is the permeability of vacuum..$^{29}$ On the other hand, based on the previous studies, ${ }^{3,6,16}$ a conducting sphere whose hemisphere is coated with a dielectric layer, the ICEP velocity of particles with the dielectric hemisphere forward is given as, 

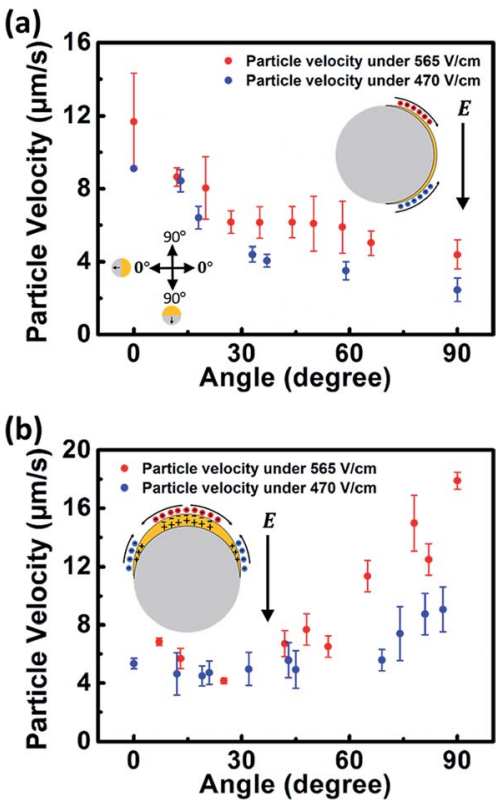

Fig. 3 (a) The relation between velocity of the magnetic Janus particles with a thin metallic coating $(10 \mathrm{~nm})$ and azimuthal angle at the field frequency of $1 \mathrm{kHz}$. Inset: (right) schematic drawing of induced charges distribution of a Janus particle with the thin metallic coating under an electric field. (Left) definition for the azimuthal angle. (b) The relation between velocity of the magnetic Janus particles with a thick metallic coating $(70 \mathrm{~nm})$ and azimuthal angle at the field frequency of $1 \mathrm{kHz}$. Inset: schematic drawing of induced charges distribution of a Janus particle with the thick metallic coating under an electric field.

$$
u=\frac{3}{64} \frac{\varepsilon a E^{2}}{\eta(1+\delta)}\left(2 \alpha^{2} \cos ^{2} \frac{\theta_{0}}{2} \sin ^{6} \frac{\theta_{0}}{2}+3 \beta^{2} \sin ^{4} \theta_{0}\right)
$$

where $\theta_{0}=\pi / 2$ for half-coating, $\alpha E$ is strength of electric field in the $\theta=0$ (or $\hat{x}$ ) direction, $\beta E$ is strength of electric field in the $\theta=$ $\pi / 2, \phi=0$ (or $\hat{y}$ ) direction, $\varepsilon$ is the permittivity, $\eta$ is the viscosity of fluid, $a$ is the particle radius, and $\delta$ is the ratio of the differential capacitances of the compact and diffuse layers. Thus, the ICEP velocity at the orientation which is normal to the field is given as, $u_{\perp}=(9 / 64)\left(\varepsilon a E^{2} / \eta(1+\delta)\right)$, and the velocity at the orientation which is parallel to the field is given as, $u_{\|}=$ $(3 / 32)\left(\varepsilon a E^{2} / \eta(1+\delta)\right)$, which is $33 \%$ less than that at the orientation normal to the field. In the case of Janus particles with a thin Au coating $(10 \mathrm{~nm})$, the polarity of the Janus particle trends to align in the direction normal to the electric field. The ICEP velocity gradually decreases with altering azimuthal angles from $0^{\circ}$ to $90^{\circ}$ and is proportional to the square of field strength (not shown in the data), as shown in Fig. 3(a). According to previous studies, this tendency results from the variation of induced-charge distribution with the orientation of particle (from normal to the electric field to parallel to the field). ${ }^{\mathbf{1 6}}$ However, from Fig. 3(a), the velocity at the orientation parallel to the field is $67 \%$ smaller than that at the orientation normal to the field. This could suggest that present ICEP theory for "dielectric coating" of the Janus particle can't satisfy the situation that a Janus particle made from a dielectric particle with half Au coating, commonly used in the experiments, moves in the direction parallel to the field due to lack of fully electric field screening ability comparing with the metal particle coated with a dielectric layer. ${ }^{27}$

To further verify the effect of thickness of coating, the motion Janus particles with a thick Au coating $(70 \mathrm{~nm})$ is measured at different orientations, which is mainly parallel to the electric field with the dielectric hemisphere forward without an external magnetic field. This could be elucidated by that, in the case of thick coating, the maximum induced dipole occurs in the direction parallel to the electric field, $\theta=90^{\circ}$. By applying the magnetic field, the ICEP velocity gradually decreases with the azimuthal angle from $90^{\circ}$ to $0^{\circ}$, as shown in Fig. 3(b). This result indicates that the strength of the induced dipole is the largest in the direction parallel to the electric field and decrease with azimuthal angle from $0^{\circ}$ to $90^{\circ}$.

The maximum ICEP velocity of a Janus particle with a thin coating is at the orientation normal to the electric field. However, in the case of thick coating, the maximum ICEP velocity is at the orientation parallel to the electric field. Therefore, the prevalent theory for ICEP couldn't adequately describe the ICEP behaviors observed in our experiments. We propose that the induced dipole of a Janus particle, depending on the thickness of coating, dominates the ICEP behavior, including the ICEO flow pattern around the particle. For instance, the induced dipole of a Janus particle with a thin coating is the largest at the orientation normal to the electric field resulting in the maximum ICEP velocity and the symmetric ICEO flow driving the motion of the particle with the dielectric forward, as shown in the inset of Fig. 3(a). On the other hand, in the case of thick coating, induced dipole is the largest at the orientation parallel to the field and results in the maximum ICEP velocity in the same direction, inset of Fig. 3(b).

Due to the hemispherical coating feature of Janus particles, the length scale of coating thickness may become significant in polarization, which is similar to modifying the length scale of a different non-spherical object under an electric field. The electrokinetics of metal nanowires (rods) is reported in the previous studies. ${ }^{30,31}$ The induced dipole on a slender metal rod can be decomposed into the long and short axes components. The induced dipole along the long axis could be written as, $p_{1}=$ $\left(4 \pi \varepsilon_{\mathrm{l}} h^{3} E_{\mathrm{h}} / 3\right)(\mathrm{i} \Omega /(1+\mathrm{i} \Omega \ln (1 / \beta)))$ and along the short axis could be written as, $p_{\mathrm{s}}=4 \pi \varepsilon_{1} r h^{2} E_{\mathrm{r}}(\mathrm{i} \Omega-1) /(\mathrm{i} \Omega+1)$, where $\varepsilon_{1}$ is the permittivity of liquid, $h$ is the half length of the long axis, $E_{\mathrm{h}}$ and $E_{\mathrm{r}}$ are the electric field along the long axis and short axis respectively, $\Omega$ is the non-dimensional frequency $\Omega=\omega C_{\mathrm{DL}} r / \sigma, r$ is the half length of short axis, $\omega$ is the frequency of the electric field, $\sigma$ is the liquid conductivity, $C$ is the capacitance of the electrical double layer and $\beta=r / h .^{31}$ The induced dipole on the slender metal rod depends on its geometry. Although the detailed analysis needs to be well constructed, the change of the coating thickness of Janus particle should alter the induced dipole and ICEP behavior of Janus particles.

\subsection{Orientation-dependent ICEO}

To investigate the dependence of ICEO flow pattern on the induced dipole, we directly measure and analyze the variation of the ICEO patterns around an immobilized particle with 


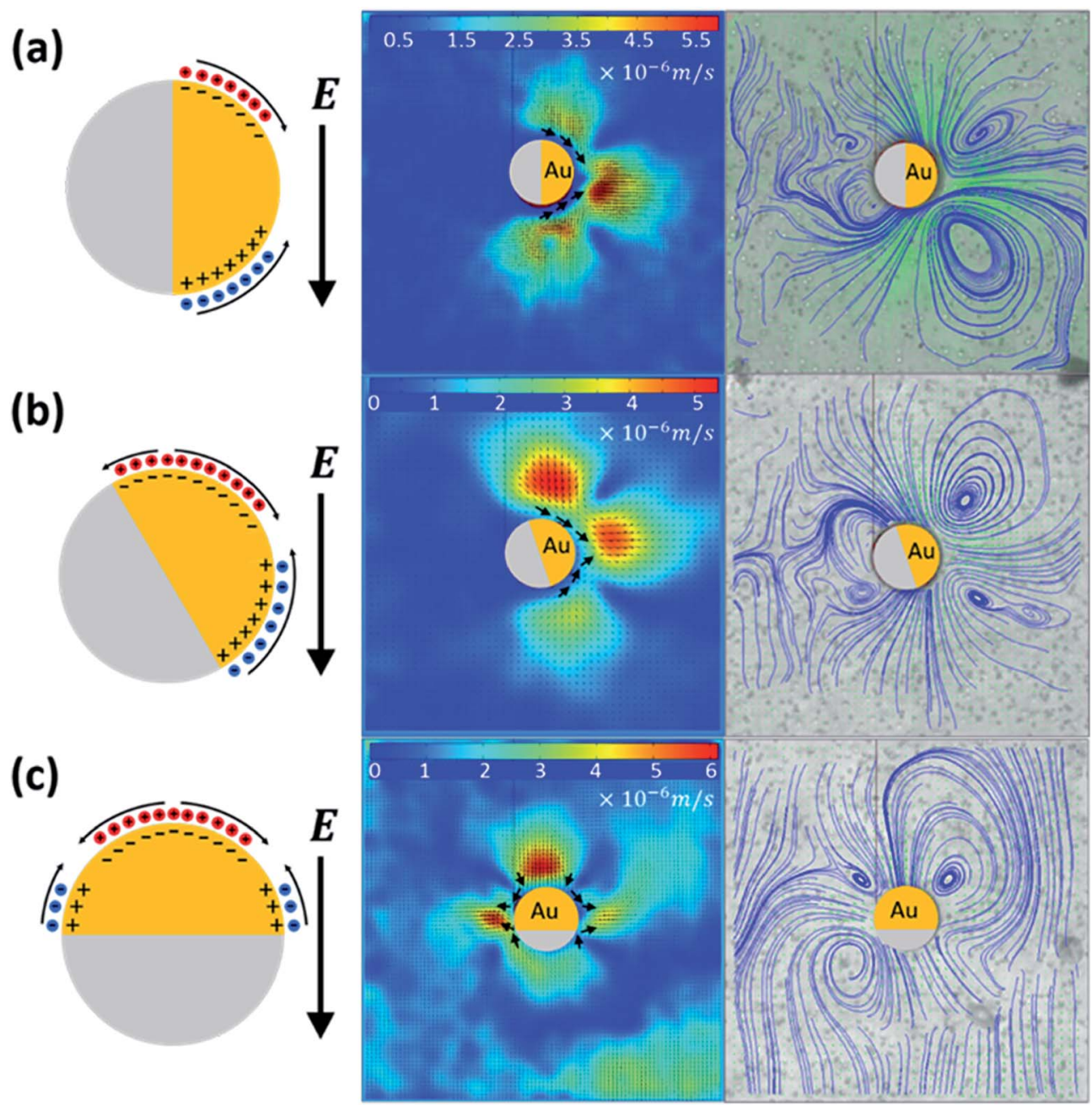

Fig. 4 The schematic drawings (left) of induced charges distribution, color maps (middle) and flow patterns (right) of ICEO around an immobilized $20 \mu \mathrm{m}$ Janus particle with $100 \mathrm{~nm}$ metallic coating at the electric field of $133.3 \mathrm{~V} \mathrm{~cm}-1$ and frequency of $1 \mathrm{kHz}$ at different azimuthal angles (orientations). (a) $0^{\circ}$, (b) $15^{\circ}$, and (c) $90^{\circ}$. Note that the density of the streamlines in the flow patterns (right) doesn't represent the flow rate, and it is just for presenting the flow patterns of ICEO.

different orientations by a particle image velocimetry (PIV) software (Matlab, PIVlab). The flow patterns of ICEO at the orientation normal to and parallel to the electric fields have been reported by Chenhui Peng et al. ${ }^{17}$ In a polar coordinate, the velocity of ICEO is given as, ${ }^{6,15,16} u_{\theta}=\left(9 a^{4} / 4 \eta\right)\left(\varepsilon a E^{2} / 1+\delta\right)$ $\cos \theta \sin \theta$, on the metal side by Bazant et al. In our experiment, when the azimuthal angle is $0^{\circ}$, the flow pattern of ICEO around the particle is a pair of significant vortices near the Au hemisphere. The velocity near the Au side is higher than that near the dielectric side due to the higher polarizability of Au coating, as shown in Fig. 4(a). By turning the azimuthal angle to $15^{\circ}$, the up-down symmetry of ICEO is broken resulting in a stronger vortex near the top of Au side, as shown in Fig. 4(b). Finally, when the azimuthal angle is $90^{\circ}$, there are two pairs of vortices appearing. One is near the $\mathrm{Au}$ side, which could drive the particle with the dielectric forward, and the other is near the PS/ $\mathrm{Au}$ interface, which could against particle moving, Fig. 4(c). The difference of the flow pattern results from the distinct distributions of the induced charges at the different orientations, as shown in the schematic drawings of Fig. 4. Note that the charge distributions in Fig. 4 are established based on the previous studies ${ }^{16}$ at different orientations of the Janus particle corresponding to a fixed electric field. This result also agrees with that the induced dipole dominates the ICEO flow around the particles.

\subsection{Trajectory control}

To investigate the stability of orientation-dependent ICEP motions, the magnet is place at the azimuthal angle $0^{\circ}$ and $90^{\circ}$, respectively. In the thin coating case, the ICEP motion of Janus particles tends to normal to the electric field (along the azimuthal angle $0^{\circ}$ ). Thus, the distribution of the ICEP moving direction is more concentrated when the magnet is place at the azimuthal angle $0^{\circ}$, as shown in Fig. 5(a) and (b). On the other hand, in the thick coating case, the ICEP motion of Janus particles tends to parallel to the electric field (along the azimuthal angle $90^{\circ}$ ). The distribution of the ICEP moving 
(a)

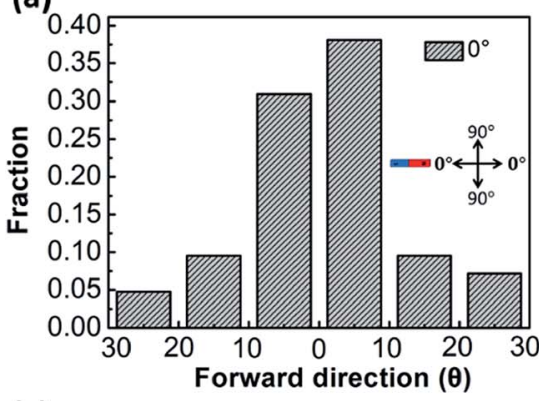

(c)

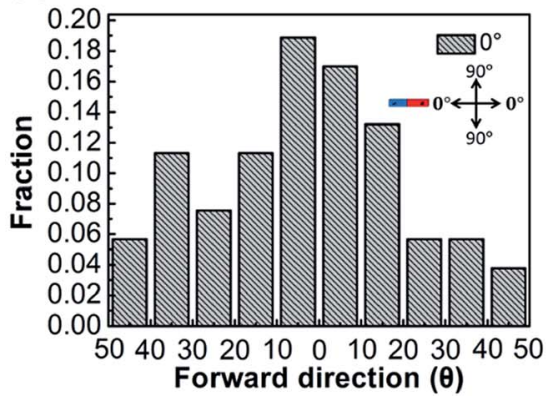

(b)

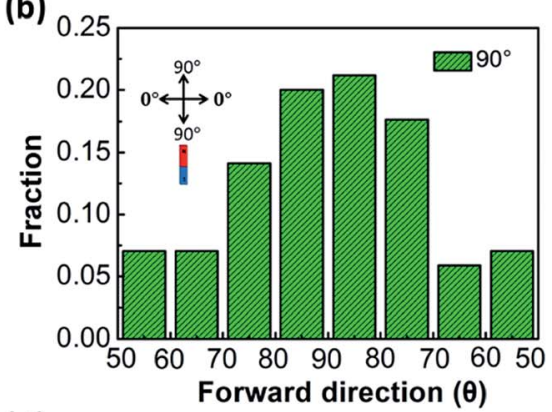

(d)

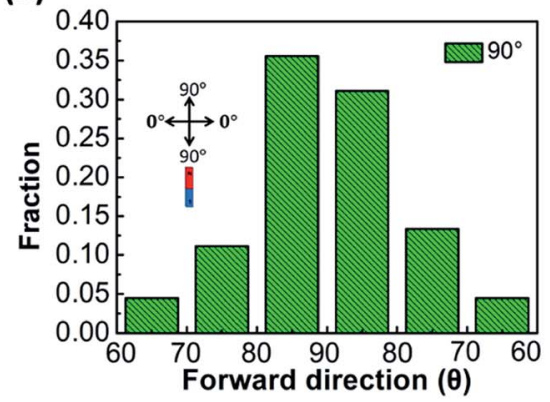

Fig. 5 The distribution of the moving direction of Janus particle. In the thin coating $(10 \mathrm{~nm})$ case, the magnet is place at the azimuthal angle (a) $0^{\circ}$, (b) $90^{\circ}$. In the thick coating $(70 \mathrm{~nm})$ case, the magnet is place at the azimuthal angle (c) $0^{\circ}$, (d) $90^{\circ}$.

direction is more concentrated when the magnet is place at the azimuthal angle $90^{\circ}$, as shown in Fig. 5(c) and (d). From Fig. 5, the ICEP trajectories of Janus particles aren't perfectly along the direction where the magnet is placed. This could result from the competition between the induced electric dipole and magnet dipole. Moreover, additional factors, such as surface roughness, contamination, and interaction with chamber wall, could result in the difference of the ICEP trajectory. ${ }^{17}$ The results from Fig. 5 further verify that the ICEP motion of Janus particles depends on the coating thickness of Janus particles.

Finally, we demonstrate that the trajectories of Janus particles under an electric field are controlled by a rotating magnetic field. In the case of the thin Au coating, the trajectories are controlled by a rotating magnetic field with different rotational speeds at a constant strength of electric field of $530 \mathrm{~V} \mathrm{~cm}^{-1}$ and frequency of $1 \mathrm{kHz}$. The result is shown in Fig. 6(a). The
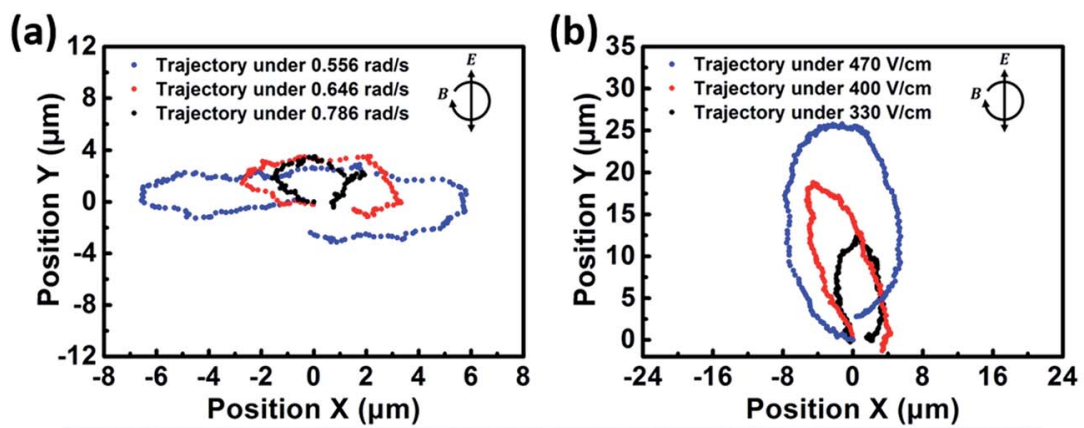

(c)

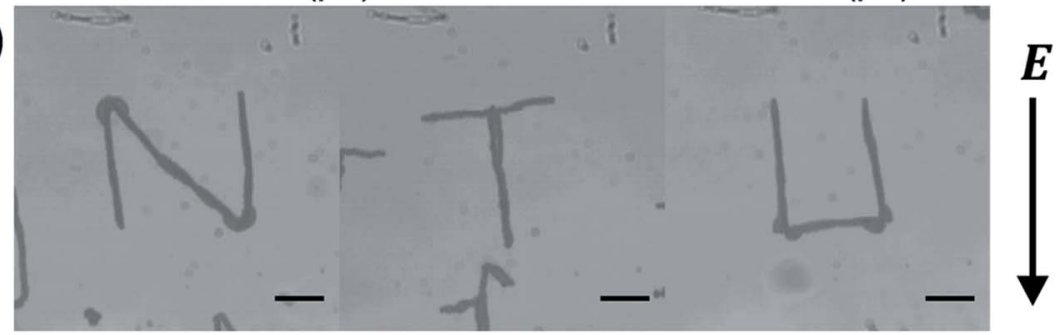

Fig. 6 (a) Trajectory control of the magnetic Janus particles with a thin metallic coating $(10 \mathrm{~nm})$ at the electric field of $530 \mathrm{~V} \mathrm{~cm}{ }^{-1}$ and frequency of $1 \mathrm{kHz}$ with different rotational speeds of a rotating magnetic field. (b) Trajectory control of the magnetic Janus particles with a thick metallic coating $(70 \mathrm{~nm})$ at the rotating magnetic field of $0.486 \mathrm{rad} \mathrm{s}^{-1}$ and frequency of $1 \mathrm{kHz}$ with different strength of electric fields. (c) The trajectory of "NTU" logo recorded from the motion of 4 particles chain. (Scale bar: $20 \mu \mathrm{m}$ ). 
trajectories are horizontally elliptical and the lengths of the trajectories are inversely proportional to rotational speed of the magnetic field. This further confirms that the Janus particle with the thin metal coating moves faster in the direction which is normal to the electric field. In the case of the thick Au coating, the trajectories are controlled with different strength of electric fields at a constant rotational speed of the magnetic field of $0.486 \mathrm{rad} \mathrm{s}^{-1}$ and frequency of $1 \mathrm{kHz}$. The trajectories are vertically elliptical and the lengths of the trajectories are proportional to the strength of electric field, which verifies that the particle with thick metal coating move faster in the direction which is parallel to the electric field, as shown in Fig. 6(b). Moreover, this study provides a programmable method to control the trajectory of magnetic Janus particles. A "NTU" logo is demonstrated by controlling the direction of the magnetic field, as shown in Fig. 6(c).

\section{Conclusions}

To conclude, we experimentally study the orientationdependent ICEP behavior of the magnetic Janus particles with different coating thicknesses at the nanoscale, where the orientation of the particles with respect to the electric field can be dynamically tuned by an external magnetic field. According to our observation, the ICEP motion of Janus particles with thin coating is normal to the electric field. However, in the thick coating case, the ICEP motion is parallel to the electric field. We propose that the strength of induced dipole of a Janus particles, depending on the thickness of metallic coating at the nanoscale and orientation of a particle, dominates the behavior of ICEP. This study may bring new insights for induced-charge electrokinetic phenomena on widely used metal coated Janus particles and give a way to control and design active materials.

\section{Conflicts of interest}

There are no conflicts to declare.

\section{Acknowledgements}

We would like to acknowledge the members of our laboratory for fruitful discussions. This work is supported through the NSC 103-2112-M-002-008-MY3 grant funded by the Ministry of Science and Technology, Taiwan, R.O.C.

\section{References}

1 H.-R. Jiang, N. Yoshinaga and M. Sano, Phys. Rev. Lett., 2010, 105, 268302.

2 J. R. Howse, R. A. Jones, A. J. Ryan, T. Gough, R. Vafabakhsh and R. Golestanian, Phys. Rev. Lett., 2007, 99, 048102.

3 S. Gangwal, O. J. Cayre, M. Z. Bazant and O. D. Velev, Phys. Rev. Lett., 2008, 100, 058302.

4 Y. Wu, X. Lin, Z. Wu, H. Möhwald and Q. He, ACS Appl. Mater. Interfaces, 2014, 6, 10476-10481.

5 F. Mou, C. Chen, Q. Zhong, Y. Yin, H. Ma and J. Guan, ACS Appl. Mater. Interfaces, 2014, 6, 9897-9903.
6 A. Ramos, P. García-Sánchez and H. Morgan, Curr. Opin. Colloid Interface Sci., 2016, 24, 79-90.

7 M. P. Hughes, Nanotechnology, 2000, 11, 124.

8 K. H. Bhatt, S. Grego and O. D. Velev, Langmuir, 2005, 21, 6603-6612.

9 D. Morganti, AC electrokinetic analysis of chemically modified microparticles, University of Southampton, 2012.

10 T. B. Jones and T. B. Jones, Electromechanics of particles, Cambridge University Press, 2005.

11 P. Sajeesh and A. K. Sen, Microfluid. Nanofluid., 2014, 17, 152.

12 A. Walther and A. H. Müller, Soft Matter, 2008, 4, 663-668.

13 M. Z. Bazant and T. M. Squires, Phys. Rev. Lett., 2004, 92, 066101.

14 T. M. Squires and M. Z. Bazant, J. Fluid Mech., 2004, 509, 217-252.

15 M. Z. Bazant, in Electrokinetics and Electrohydrodynamics in Microsystems, Springer, 2011, pp. 221-297.

16 T. M. Squires and M. Z. Bazant, J. Fluid Mech., 2006, 560, 65101.

17 C. Peng, I. Lazo, S. V. Shiyanovskii and O. D. Lavrentovich, Phys. Rev. E: Stat., Nonlinear, Soft Matter Phys., 2014, 90, 051002.

18 A. Boymelgreen, G. Yossifon and T. Miloh, Langmuir, 2016, 32, 9540-9547.

19 R. Suzuki, H.-R. Jiang and M. Sano, arXiv preprint arXiv:1104.5607, 2011.

20 Y. Daghighi, I. Sinn, R. Kopelman and D. Li, Electrochim. Acta, 2013, 87, 270-276.

21 L. Baraban, D. Makarov, O. G. Schmidt, G. Cuniberti, P. Leiderer and A. Erbe, Nanoscale, 2013, 5, 1332-1336.

22 L. Baraban, D. Makarov, R. Streubel, I. Mönch, D. Grimm, S. Sanchez and O. G. Schmidt, ACS Nano, 2012, 6, 3383-3389.

23 A. Ruditskiy, B. Ren and I. Kretzschmar, Soft Matter, 2013, 9, 9174-9181.

24 B. Ren, A. Ruditskiy, J. H. Song and I. Kretzschmar, Langmuir, 2011, 28, 1149-1156.

25 S. K. Smoukov, S. Gangwal, M. Marquez and O. D. Velev, Soft Matter, 2009, 5, 1285-1292.

26 Y. L. Chen and H. R. Jiang, J. Visualized Exp., 2017, DOI: 10.3791/55950.

27 Y.-L. Chen and H.-R. Jiang, Appl. Phys. Lett., 2016, 109, 191605.

28 S. Ebbens, D. Gregory, G. Dunderdale, J. Howse, Y. Ibrahim, T. Liverpool and R. Golestanian, EPL, 2014, 106, 58003.

29 S. S. Shevkoplyas, A. C. Siegel, R. M. Westervelt, M. G. Prentiss and G. M. Whitesides, Lab Chip, 2007, 7, 1294-1302.

30 J. J. Arcenegui, P. García-Sánchez, H. Morgan and A. Ramos, Phys. Rev. E: Stat., Nonlinear, Soft Matter Phys., 2013, 88, 033025.

31 J. J. Arcenegui, P. García-Sánchez, H. Morgan and A. Ramos, Phys. Rev. E: Stat., Nonlinear, Soft Matter Phys., 2013, 88, 063018. 